\title{
Asymmetric Transfer Hydrogenation of Ketones Catalyzed by Hydrophobic Metal-Amido Complexes in Aqueous Micelles and Vesicles
}

Fei Wang, ${ }^{a, b}$ Hui Liu, ${ }^{a}$ Linfeng Cun, ${ }^{a}$ Jin Zhu, ${ }^{* a, c}$ Jingen Deng, ${ }^{* a, b}$ and Yaozhong Jiang ${ }^{a}$

${ }^{a}$ Key Laboratory of Asymmetric Synthesis \& Chirotechnology of Sichuan Province and Union

Laboratory of Asymmetric Synthesis, Chengdu Institute of Organic Chemistry, Chinese Academy of

Sciences, Chengdu 610041, China. ${ }^{b}$ Graduate School of Chinese Academy of Sciences, Beijing, China.

${ }^{c}$ State Key Laboratory of Applied Organic Chemistry, Lanzhou University, Lanzhou 730000, China

jgdeng@cioc.ac.cn

Supporting Information

Table of Contents

1. General methods .52

2. General procedure and results of asymmetric transfer hydrogenation of ketones.............S2-S3

3. Optical rotation, NMR data and GC conditions for 3a, 3c, 5 and 13-19...........S4-S6

4. Recycle experiment for asymmetric transfer hydrogenation of acetophenone ........S7-S7

5. General procedure for asymmetric transfer hydrogenation of $\alpha$-bromomethylaromatic

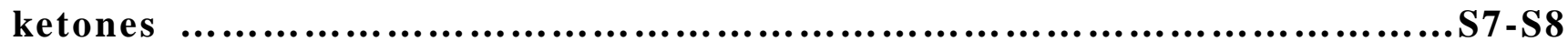

6. Optical rotation, NMR data and GC or HPLC conditions for 21a-21g, 22a and 23a........S8-S11

7. References...........................................................................................S11 
General methods. Melting points were determined in open capillaries and were uncorrected. The NMR spectra were recorded with tetramethylsilane $(\delta 0.00)$ as the internal standard. Enantiomeric excess was determined by GC on chiral CP-cyclodex B-236 M/ CP-Chirasil-DEX CB columns or HPLC on Chiral $\mathrm{OD} / \mathrm{OJ}$ columns. The concentration of ruthenium in both organic and aqueous layers was determined by ICP-MS analysis. The $\alpha$-bromomethylaromatic ketones were prepared from the corresponding ketones as reported in the literature. ${ }^{1}(R, R)$-18-Electron Ruthenium complex (1a) was prepared as reported in the literature. ${ }^{2}$ All other reagents were used without purification as commercially available. CTAB $=$ Cetyltrimethylammonium bromide; SDS = Sodium dodecyl sulfate; Triton X-100 = Polyethylene glycol mono [4-(1,1,3,3-tetramethylbutyl)phenyl] ether; DDAPS $=$ Dodecyldimethylammonio propanesulfonate.

General procedure for asymmetric transfer hydrogenation of ketones. $(R, R)-\mathrm{Ts} D P E N-\mathrm{Ru}(1 \mathrm{a})$ (1.3 mg, $0.002 \mathrm{mmol})$, surfactant $(0.02 \mathrm{mmol})$, ketones $(0.2 \mathrm{mmol})$ and $\mathrm{HCO}_{2} \mathrm{Na} \cdot 2 \mathrm{H}_{2} \mathrm{O}(104 \mathrm{mg}, 1.0$ mmol) were added into degassed water $(0.5 \mathrm{~mL})$. The mixture was degassed for three times and stirred at $28{ }^{\circ} \mathrm{C}$ under an argon atmosphere with $800 \mathrm{r} / \mathrm{min}$. When the reaction was completed (determined by TLC), the reaction mixture was extracted with $\mathrm{CH}_{2} \mathrm{Cl}_{2}(5 \mathrm{~mL})$ for three times. The conversion and enantioselectivity were determined by GC analysis. Isolated yield was obtained by flash chromatography.

TABLE 1. Asymmetric Transfer Hydrogenation of Acetophenone (2a) and 1-Indanone (4) in Aqueous Media with Different Concentration of CTAB ${ }^{\mathrm{a}}$ 


\begin{tabular}{|c|c|c|c|c|c|c|c|}
\hline Entry & Concentration & $\operatorname{Conv}(\mathbf{2 a})^{b}$ & $\operatorname{TOF}(\mathbf{2} \mathbf{a})^{\mathrm{c}}$ & $\operatorname{Ee}(\mathbf{3 a})^{b}$ & $\operatorname{Conv}(4)^{b}$ & $\operatorname{TOF}(\mathbf{4})^{\mathrm{c}}$ & $\operatorname{Ee}(\mathbf{5})^{b}$ \\
\hline & (M) & $\%$ & $\left(\mathrm{~h}^{-1}\right)$ & $\%$ & $\%$ & $\left(h^{-1}\right)$ & $\%$ \\
\hline 1 & 0 & 51.9 & 51.9 & 94.3 & 37.9 & 18.9 & 96.8 \\
\hline 2 & 0.004 & 60.9 & 60.9 & 95.2 & 53.2 & 26.6 & 97.2 \\
\hline 3 & 0.02 & 66.0 & 66.0 & 95.7 & 60.2 & 30.1 & 97.3 \\
\hline 4 & 0.04 & 68.5 & 68.5 & 95.4 & 61.9 & 31.0 & 98.1 \\
\hline 5 & 0.08 & 53.1 & 53.1 & 95.0 & 50.0 & 25.0 & 98.1 \\
\hline 6 & 0.12 & 45.2 & 45.2 & 95.1 & 46.7 & 23.4 & 98.5 \\
\hline 7 & 0.2 & 38.7 & 38.7 & 95.3 & 31.8 & 15.9 & 98.0 \\
\hline 8 & 0.24 & 30.5 & 30.5 & 93.5 & 27.1 & 13.5 & 98.4 \\
\hline 9 & 0.32 & 22.1 & 22.1 & 92.9 & 26.1 & 13.0 & 98.3 \\
\hline 10 & 0.4 & 17.0 & 17.0 & 92.8 & 27.8 & 13.9 & 98.3 \\
\hline 11 & 0.6 & 9.7 & 9.7 & 89.4 & 19.2 & 9.6 & 98.1 \\
\hline
\end{tabular}

${ }^{\mathrm{a}}[\mathrm{TsDPEN}-\mathrm{Ru}]=0.004 \mathrm{M} ;[\mathrm{TsDPEN}-\mathrm{Ru}]:[\mathrm{CTAB}]:[$ Substrate $]:\left[\mathrm{HCO}_{2} \mathrm{Na}\right]=1: 10: 100: 500 ; 0.5 \mathrm{~mL}$ of degassed water; under an argon atmosphere; $28{ }^{\circ} \mathrm{C}$. ${ }^{\mathrm{b}}$ The conversion and ee were determined by GC analysis (CP-cyclodex B-236 M). ${ }^{\mathrm{c}}$ The average TOFs were calculated over the 1 hour for $\mathbf{2 a}$ and 2 hours for 4 . 


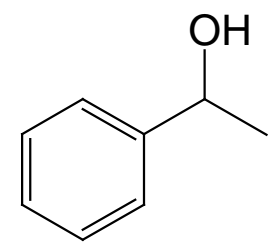

(R)-1-Phenylethanol (3a): $[\alpha]_{\mathrm{D}}{ }^{23}+51.2^{\circ}\left(c\right.$ 1.10, $\left.\mathrm{CH}_{2} \mathrm{Cl}_{2}\right) ; 95.1 \%$ ee; ${ }^{1} \mathrm{H}-\mathrm{NMR}$ $\left(\mathrm{CDCl}_{3}, 300 \mathrm{MHz}\right), \delta: 7.42-7.30(\mathrm{~m}, 5 \mathrm{H}), 4.94(\mathrm{q}, J=6.3 \mathrm{~Hz}, 1 \mathrm{H}), 1.52(\mathrm{~d}, J=6.3 \mathrm{~Hz}$, 3H) ppm; GC analysis: CP-cyclodex B-236 M, $0.25 \mathrm{~mm} \times 25 \mathrm{~m}$, column temperature $=$ $115{ }^{\circ} \mathrm{C}$ (isothermal), inject temperature $=220{ }^{\circ} \mathrm{C}$, detector temperature $=240{ }^{\circ} \mathrm{C}$, inlet pressure $=10.5 \mathrm{psi}$; $\mathrm{t}_{\text {major }}=5.0 \mathrm{~min}, \mathrm{t}_{\text {minor }}=5.3 \mathrm{~min}$.

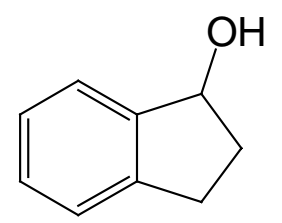

(R)-1-Indanol (5): $[\alpha]_{\mathrm{D}}{ }^{23}-35.1^{\circ}\left(c 1.12, \mathrm{CHCl}_{3}\right) ; 98.2 \%$ ee; ${ }^{1} \mathrm{H}-\mathrm{NMR}\left(\mathrm{CDCl}_{3}, 300\right.$

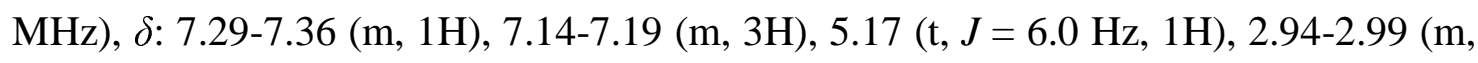
1H), 2.75-2.78 (m, 1H), 2.40-2.42 (m, 1H), 1.84-1.91 (m, 1H) ppm; GC analysis: CP-Chirasil-DEX CB, $0.25 \mathrm{~mm} \times 25 \mathrm{~m}$; column temperature $=130{ }^{\circ} \mathrm{C}$, inject temperature $=220{ }^{\circ} \mathrm{C}$, detector temperature $=240$ ${ }^{\circ} \mathrm{C}$, inlet pressure $=11.5 \mathrm{psi} ; \mathrm{t}_{\text {minor }}=9.9 \mathrm{~min}, \mathrm{t}_{\text {major }}=10.2 \mathrm{~min}$.

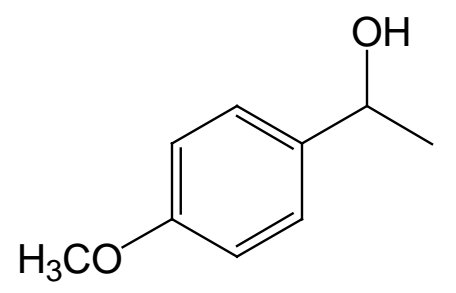

$(\boldsymbol{R})-1-(p-M e t h o x y p h e n y l) e t h a n o l(3 c):[\alpha]_{\mathrm{D}}{ }^{23}=+44.2^{\circ}\left(c 1.2, \mathrm{CHCl}_{3}\right) ; 94.5 \%$ ee. ${ }^{1} \mathrm{H}$ NMR (300 MHz, $\left.\mathrm{CDCl}_{3}\right), \delta: 7.29(\mathrm{~d}, J=7.4 \mathrm{~Hz}, 2 \mathrm{H}), 6.88(\mathrm{~d}, J=7.4$ $\mathrm{Hz}, 2 \mathrm{H}), 4.84(\mathrm{q}, J=6.4 \mathrm{~Hz}, 1 \mathrm{H}), 3.80(\mathrm{~s}, 3 \mathrm{H}), 1.90(\mathrm{br}, 1 \mathrm{H}), 1.47$ (d, $J=6.4$

$\mathrm{Hz}, 3 \mathrm{H}) \mathrm{ppm}$; GC analysis: CP-cyclodex B-236 M, $0.25 \mathrm{~mm} \times 25 \mathrm{~m}$, column temperature $=140{ }^{\circ} \mathrm{C}$ (isothermal), inject temperature $=240{ }^{\circ} \mathrm{C}$, detector temperature $=260{ }^{\circ} \mathrm{C}$, inlet pressure $=10 \mathrm{psi} ; \mathrm{t}_{\text {major }}=$ $5.1 \mathrm{~min}, \mathrm{t}_{\text {minor }}=5.4 \mathrm{~min}$. 


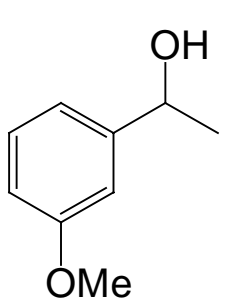

(R)-1-(m-Methoxyphenyl)ethanol (13): $[\alpha]_{\mathrm{D}}{ }^{23}=+31.8^{\circ}\left(c \quad 1.1, \mathrm{CHCl}_{3}\right) ; 94.0 \%$ ee. ${ }^{1} \mathrm{H}$ NMR (300 MHz, $\left.\mathrm{CDCl}_{3}\right), \delta:$ 7.39-7.33 (m, 1H), 7.05-7.03 (m, 2H), 6.93-6.89 (m, 1H), $4.93(\mathrm{q}, J=6.4 \mathrm{~Hz}, 1 \mathrm{H}), 3.91(\mathrm{~s}, 3 \mathrm{H}), 1.70(\mathrm{br}, 1 \mathrm{H}), 1.57(\mathrm{~d}, J=6.5 \mathrm{~Hz}, 3 \mathrm{H}) \mathrm{ppm} ; \mathrm{GC}$ analysis: CP-cyclodex B-236 M, $0.25 \mathrm{~mm} \times 25 \mathrm{~m}$, column temperature $=150{ }^{\circ} \mathrm{C}$ (isothermal), inject temperature $=240{ }^{\circ} \mathrm{C}$, detector temperature $=260{ }^{\circ} \mathrm{C}$, inlet pressure $=10 \mathrm{psi} ; \mathrm{t}_{\text {major }}=$ $4.4 \mathrm{~min}, \mathrm{t}_{\mathrm{minor}}=4.6 \mathrm{~min}$.

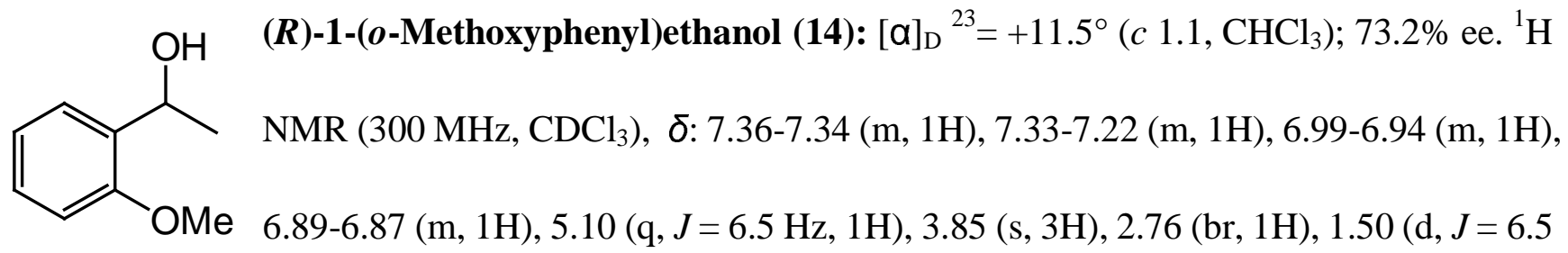

$\mathrm{Hz}, 3 \mathrm{H}) \mathrm{ppm}$; GC analysis: CP-cyclodex B-236 M, $0.25 \mathrm{~mm} \times 25 \mathrm{~m} \sqcap$ column temperature $=140{ }^{\circ} \mathrm{C}$ (isothermal), inject temperature $=240{ }^{\circ} \mathrm{C}$, detector temperature $=260{ }^{\circ} \mathrm{C}$, inlet pressure $=12 \mathrm{psi} ; \mathrm{t}_{\text {minor }}=$ $4.5 \mathrm{~min}, \mathrm{t}_{\mathrm{major}}=4.8 \mathrm{~min}$.

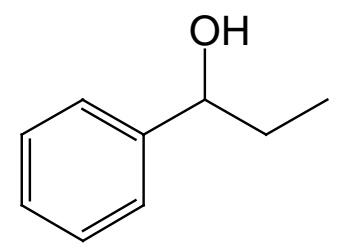

(R)-1-Phenylpropan-1-ol (15): $[\alpha]_{\mathrm{D}}{ }^{23}=+37.1^{\circ}\left(c 0.9, \mathrm{CHCl}_{3}\right) ; 87.9 \%$ ee. ${ }^{1} \mathrm{H}-\mathrm{NMR}$ $\left(\mathrm{CDCl}_{3}, 300 \mathrm{MHz}\right), \delta .7 .48-7.39(\mathrm{~m}, 5 \mathrm{H}), 4.73(\mathrm{t}, J=6.5 \mathrm{~Hz}, 1 \mathrm{H}), 1.96-1.88(\mathrm{~m}, 2 \mathrm{H})$, $1.05(\mathrm{t}, J=7.5 \mathrm{~Hz}, 3 \mathrm{H}) \mathrm{ppm}$; GC analysis: CP-Chirasil-DEX CB, $0.25 \mathrm{~mm} \times 25 \mathrm{~m}$, column temprature $=140{ }^{\circ} \mathrm{C}$ (isothermal), inject temprature $=240{ }^{\circ} \mathrm{C}$, detector temprature $=260{ }^{\circ} \mathrm{C}$, inlet pressure $=11 \mathrm{psi} ; \mathrm{t}_{\text {major }}=4.8 \mathrm{~min}, \mathrm{t}_{\text {minor }}=5.0 \mathrm{~min}$.<smiles>CC(C)C(O)c1ccccc1</smiles>

(R)-2-Methyl-1-phenylpropan-1-ol (16): $[\alpha]_{\mathrm{D}}{ }^{23}=+12.3^{\circ}\left(c 1.2, \mathrm{CHCl}_{3}\right) ; 38.3 \%$ ee.

${ }^{1} \mathrm{H}$ NMR $\left(300 \mathrm{MHz}, \mathrm{CDCl}_{3}\right), \delta: 7.37-7.25(\mathrm{~m}, 5 \mathrm{H}), 4.36(\mathrm{~d}, J=6.9 \mathrm{~Hz}, 1 \mathrm{H})$, 
2.01-1.88 (m, 1H), $1.00(\mathrm{~d}, J=6.7 \mathrm{~Hz}, 3 \mathrm{H}), 0.79(\mathrm{~d}, J=6.7 \mathrm{~Hz}, 3 \mathrm{H}) \mathrm{ppm}$; GC analysis: CP-cyclodex B-236 M, $0.25 \mathrm{~mm} \times 25 \mathrm{~m}$, column temperature $=150{ }^{\circ} \mathrm{C}$ (isothermal), inject temperature $=240{ }^{\circ} \mathrm{C}$, detector temperature $=260{ }^{\circ} \mathrm{C}$, inlet pressure $=11 \mathrm{psi} ; \mathrm{t}_{\text {major }}=17.5 \mathrm{~min}, \mathrm{t}_{\text {minor }}=18.6 \mathrm{~min}$.

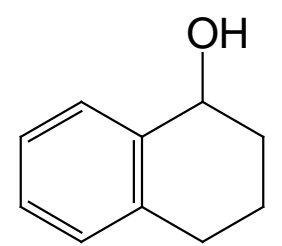

$(\boldsymbol{R})-\mathbf{1 , 2 , 3 , 4 - T e t r a h y d r o - 1 - n a p h t h o l ~ ( 1 7 ) : ~}[\alpha]_{\mathrm{D}}^{23}=-32.5^{\circ}\left(c \quad 1.0, \mathrm{CHCl}_{3}\right) ; 96.9 \%$ ee. ${ }^{1} \mathrm{H}-\mathrm{NMR}\left(\mathrm{CDCl}_{3}, 300 \mathrm{MHz}\right), \delta:$ 7.45-7.42 (m, 1H), 7.26-7.19 (m, 2H), 7.13-7.11 (m, $1 \mathrm{H}), 4.78(\mathrm{t}, J=5.0 \mathrm{~Hz}, 1 \mathrm{H}), 2.87-2.75(\mathrm{~m}, 2 \mathrm{H}), 2.02-1.79(\mathrm{~m}, 5 \mathrm{H}) \mathrm{ppm}$; $\mathrm{GC}$ analysis: CP-cyclodex B-236 M, $0.25 \mathrm{~mm} \times 25 \mathrm{~m}$, column temprature $=130{ }^{\circ} \mathrm{C}$ (isothermal), inject temprature $=$ $220{ }^{\circ} \mathrm{C}$, detector temprature $=260{ }^{\circ} \mathrm{C}$, inlet pressure $=11 \mathrm{psi} ; \mathrm{t}_{\text {minor }}=13.3 \mathrm{~min}, \mathrm{t}_{\text {major }}=13.6 \mathrm{~min}$.

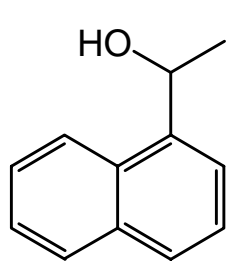

(R)-1-(1'-Naphthyl)ethanol (18): $[\alpha]_{\mathrm{D}}{ }^{23}=+69.7^{\circ}\left(c\right.$ 1.1, $\left.\mathrm{Et}_{2} \mathrm{O}\right)$; $90.4 \%$ ee. ${ }^{1} \mathrm{H}$ NMR (300 MHz, $\left.\mathrm{CDCl}_{3}\right), \delta: 8.12-8.08(\mathrm{~m}, 1 \mathrm{H}), 7.91-7.88(\mathrm{~m}, 1 \mathrm{H}), 7.84(\mathrm{~d}, J=8.2 \mathrm{~Hz}, 1 \mathrm{H})$, $7.66(\mathrm{~d}, J=7.1 \mathrm{~Hz}, 1 \mathrm{H}), 7.56-7.45(\mathrm{~m}, 3 \mathrm{H}), 5.62(\mathrm{q}, J=6.5 \mathrm{~Hz}, 1 \mathrm{H}), 2.37(\mathrm{br}, 1 \mathrm{H}), 1.65$ $(\mathrm{d}, J=6.5 \mathrm{~Hz}, 3 \mathrm{H}) \mathrm{ppm} ; \mathrm{GC}$ analysis: CP-cyclodex B-236 M, $0.25 \mathrm{~mm} \times 25 \mathrm{~m}$, column temperature $=$ $160{ }^{\circ} \mathrm{C}$ (isothermal), inject temperature $=240{ }^{\circ} \mathrm{C}$, detector temperature $=260{ }^{\circ} \mathrm{C}$, inlet pressure $=11.5 \mathrm{psi}$; $\mathrm{t}_{\text {minor }}=11.2 \mathrm{~min}, \mathrm{t}_{\text {major }}=12.0 \mathrm{~min}$.

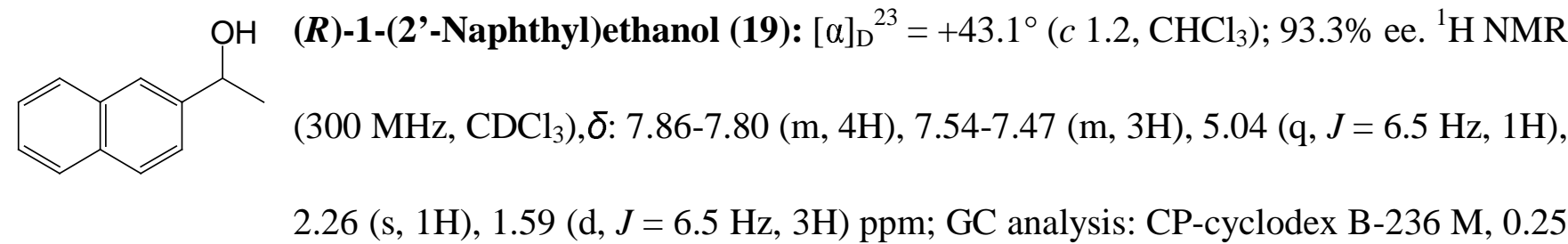

$\mathrm{mm} \times 25 \mathrm{~m}$, column temperature $=170{ }^{\circ} \mathrm{C}$ (isothermal), inject temperature $=240{ }^{\circ} \mathrm{C}$, detector temperature $=260{ }^{\circ} \mathrm{C}$, inlet pressure $=12 \mathrm{psi} ; \mathrm{t}_{\text {major }}=5.0 \mathrm{~min}, \mathrm{t}_{\text {minor }}=5.2 \mathrm{~min}$. 
Recycle experiment for asymmetric transfer hydrogenation of acetophenone (2a). $(R, R)$-TsDPEN-Ru (1a) $(1.3 \mathrm{mg}, 0.002 \mathrm{mmol}), \mathrm{CTAB}(21.9 \mathrm{mg}, 0.06 \mathrm{mmol})$, acetophenone $(24 \mu \mathrm{L}, 0.2$ mmol) and $\mathrm{HCO}_{2} \mathrm{Na} \cdot 2 \mathrm{H}_{2} \mathrm{O}(104 \mathrm{mg}, 1 \mathrm{mmol})$ were added into degassed water $(0.5 \mathrm{~mL})$. The mixture was degassed for three times and stirred at $28^{\circ} \mathrm{C}$ under an argon atmosphere with $800 \mathrm{r} / \mathrm{min}$. After the reaction was completed (determined by $\mathrm{TLC}), \mathrm{HCO}_{2} \mathrm{H}(9 \mu \mathrm{L}, 0.22 \mathrm{mmol})$ was added to regenerate sodium formate, and the product was extracted with $n$-hexane $(1 \mathrm{~mL})$ for three times. Acetophenone $(24 \mu \mathrm{L}, 0.2$ mmol) was added into the aqueous solution for a new reaction cycle, and then the mixture was degassed for three times and stirred at $28^{\circ} \mathrm{C}$ under an argon atmosphere with $800 \mathrm{r} / \mathrm{min}$. The conversion and enantioselectivity were determined by GC analysis.

\section{FIGURE 1.}

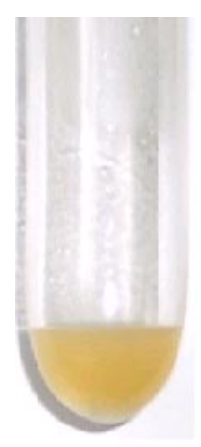

(a)

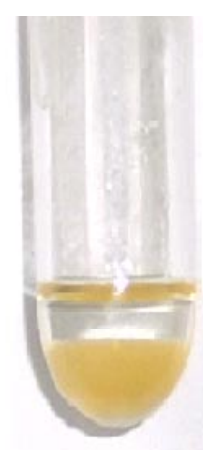

(b)

(a) Reaction mixture of acetophenone (2a) and hydrophobic catalyst (1a) in water in the presence of 0.12 M CTAB. (b) Separated phases after extracted with $n$-hexane.

General procedure for asymmetric transfer hydrogenation of $\alpha$-bromomethylaromatic ketones. The mixture of $(R, R)$-TsDPEN $(1.6 \mathrm{mg}, 0.004 \mathrm{mmol})$ and $\left[\mathrm{RhCl}_{2}(\mathrm{cp} *)\right]_{2}(1.3 \mathrm{mg}, 0.002 \mathrm{mmol})$ was added to a flask and degassed for three times. $\mathrm{CH}_{2} \mathrm{Cl}_{2}(0.5 \mathrm{~mL})$ was added to dissolve the mixture and the solution was stirred at $40^{\circ} \mathrm{C}$ for 1 hour. After removal of $\mathrm{CH}_{2} \mathrm{Cl}_{2}$ under reduced pressure, a mixture of 
$\alpha$-bromomethylaromatic ketones $(0.4 \mathrm{mmol})$, surfactant $(0.04 \mathrm{mmol}), \mathrm{HCO}_{2} \mathrm{Na} \cdot 2 \mathrm{H}_{2} \mathrm{O}(208 \mathrm{mg}, 2 \mathrm{mmol})$ and $1 \mathrm{~mL}$ of water were added. After degassed for three times, the reaction mixture was stirred at $28^{\circ} \mathrm{C}$ under an argon atmosphere with $800 \mathrm{r} / \mathrm{min}$. When the reaction was completed (determined by TLC), the reaction mixture was extracted with $\mathrm{CH}_{2} \mathrm{Cl}_{2}(5 \mathrm{~mL})$ for three times. The combined organic phases were dried over anhydrous $\mathrm{Na}_{2} \mathrm{SO}_{4}$ and evaporated under reduced pressure. The crude product was dissolved in $2 \mathrm{~mL}$ of $\mathrm{CDCl}_{3}$ and the yield was determined by ${ }^{1} \mathrm{H}-\mathrm{NMR}$ analysis using 1,3,5-trimethylbenzene as an internal standard. Isolated yield was obtained by flash chromatography.

\section{Optical rotation, NMR data and GC or HPLC conditions for 21a-21g, 22a and 23a.}

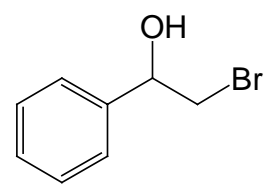

(S)-2-Bromo-1-phenylethanol (21a): $[\alpha]_{\mathrm{D}}^{23}+52.3^{\circ}\left(c \quad 1.00, \mathrm{CHCl}_{3}\right) ; 98.0 \%$ ee. ${ }^{1} \mathrm{H}-\mathrm{NMR}\left(\mathrm{CDCl}_{3}, 300 \mathrm{M} \mathrm{Hz}\right), \delta$ : 7.31-7.20 (m, 5H), $4.93(\mathrm{dd}, J=3.3,8.8 \mathrm{~Hz}, 1 \mathrm{H}), 3.56$ $(\mathrm{dd}, J=3.4,10.4 \mathrm{~Hz}, 1 \mathrm{H}), 3.46(\mathrm{dd}, J=8.9,10.4 \mathrm{~Hz}, 1 \mathrm{H}), 2.63$ (br, 1H) ppm; GC analysis: CP-cyclodex B-236 M, $0.25 \mathrm{~mm} \times 25 \mathrm{~m}$, column temperature $=130{ }^{\circ} \mathrm{C}$ (isothermal), inject temperature $=200{ }^{\circ} \mathrm{C}$, detector temperature $=220{ }^{\circ} \mathrm{C}$, inlet pressure $=10 \mathrm{psi} ; \mathrm{t}_{\text {major }}=17.4 \mathrm{~min}, \mathrm{t}_{\text {minor }}=18.2 \mathrm{~min}$.

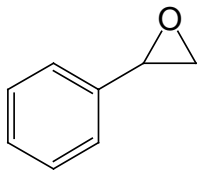

(S)-Phenyloxirane (22a): $[\alpha]_{\mathrm{D}}{ }^{23}+25.1^{\circ}\left(c 1.10, \mathrm{CHCl}_{3}\right) ; 97.2 \%$ ee. ${ }^{1} \mathrm{H}-\mathrm{NMR}\left(\mathrm{CDCl}_{3}\right.$, $300 \mathrm{MHz}), \delta .7 .38-7.28(\mathrm{~m}, 5 \mathrm{H}), 3.88(\mathrm{dd}, J=2.6,4.0 \mathrm{~Hz}, 1 \mathrm{H}), 3.16(\mathrm{dd}, J=4.1,5.5 \mathrm{~Hz}$, 1H), $2.82(\mathrm{dd}, J=2.6,5.5 \mathrm{~Hz}, 1 \mathrm{H})$ ppm; GC analysis: CP-cyclodex B-236 M, $0.25 \mathrm{~mm} \times 25 \mathrm{~m}$, column temperature $=90{ }^{\circ} \mathrm{C}$ (isothermal), inject temperature $=200{ }^{\circ} \mathrm{C}$, detector temperature $=220{ }^{\circ} \mathrm{C}$, inlet pressure $=10 \mathrm{psi} ; \mathrm{t}_{\text {major }}=7.0 \mathrm{~min}, \mathrm{t}_{\text {minor }}=7.3 \mathrm{~min}$. 


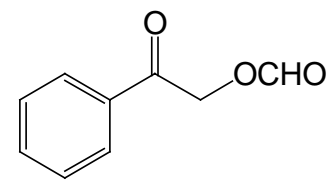

Phenacyl formate (23a): ${ }^{1} \mathrm{H}-\mathrm{NMR}\left(\mathrm{CDCl}_{3}, 300 \mathrm{M} \mathrm{Hz}\right), \delta .8 .18(\mathrm{~s}, 1 \mathrm{H}), 7.85(\mathrm{dd}, J=$ $1.2,8.6 \mathrm{~Hz}, 2 \mathrm{H}), 7.58-7.52(\mathrm{~m}, 1 \mathrm{H}), 7.45-7.39(\mathrm{~m}, 2 \mathrm{H}), 5.38(\mathrm{~s}, 2 \mathrm{H}) \mathrm{ppm}$.

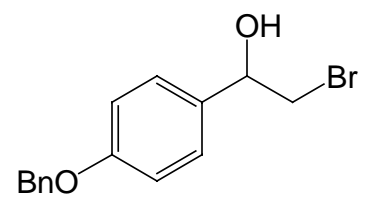

(S)-2-Bromo-1-(4-benzyloxyphenyl)ethanol (21b): $[\alpha]_{\mathrm{D}}{ }^{23}+29.7^{\circ}(c) 1.05$, $\left.\mathrm{CHCl}_{3}\right) ; 94.8 \%$ ee. ${ }^{1} \mathrm{H}-\mathrm{NMR}\left(\mathrm{CDCl}_{3}, 300 \mathrm{MHz}\right), \delta .7 .44-7.29(\mathrm{~m}, 7 \mathrm{H}), 6.98(\mathrm{dd}, J$ $=2.1,6.7 \mathrm{~Hz}, 2 \mathrm{H}), 5.07(\mathrm{~s}, 2 \mathrm{H}), 4.88(\mathrm{dd}, J=3.9,9.0 \mathrm{~Hz}, 1 \mathrm{H}), 3.60(\mathrm{dd}, J=3.6,10.4 \mathrm{~Hz}, 1 \mathrm{H}), 3.53(\mathrm{dd}, J$ = 9.2, $10.1 \mathrm{~Hz}, 1 \mathrm{H}), 1.72(\mathrm{br}, 1 \mathrm{H}) \mathrm{ppm}$; HPLC analysis: Chiralcel OD, $i-\mathrm{PrOH} / n$-hexane = 10/90 (v/v), $1.0 \mathrm{~mL} / \mathrm{min}, 23{ }^{\circ} \mathrm{C}$, UV $230 \mathrm{~nm} ; \mathrm{t}_{\text {major }}=13.8 \mathrm{~min}, \mathrm{t}_{\mathrm{minor}}=15.7 \mathrm{~min}$.

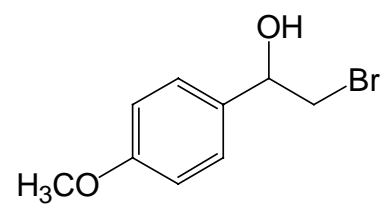

(S)-2-Bromo-1-(4-methoxyphenyl)ethanol (21c): $[\alpha]_{\mathrm{D}}^{23}+36.7^{\circ}(c$ 1.03, $\left.\mathrm{CHCl}_{3}\right) ; 97.5 \%$ ee. ${ }^{1} \mathrm{H}-\mathrm{NMR}\left(\mathrm{CDCl}_{3}, 300 \mathrm{MHz}\right), \delta .7 .23(\mathrm{~d}, J=8.6 \mathrm{~Hz}, 2 \mathrm{H}), 6.83$ $(\mathrm{d}, J=8.6 \mathrm{~Hz}, 2 \mathrm{H}), 4.80(\mathrm{dd}, J=3.8,8.8 \mathrm{~Hz}, 1 \mathrm{H}), 3.73(\mathrm{~s}, 3 \mathrm{H}), 3.52(\mathrm{dd}, J=3.6,10.4 \mathrm{~Hz}, 1 \mathrm{H}), 3.44(\mathrm{dd}$, $J=8.2,10.4 \mathrm{~Hz}, 1 \mathrm{H}), 2.53(\mathrm{br}, 1 \mathrm{H}) \mathrm{ppm}$; GC analysis: CP-cyclodex B-236 M, $0.25 \mathrm{~mm} \times 25 \mathrm{~m}$, column temperature $=160{ }^{\circ} \mathrm{C}$ (isothermal), inject temperature $=220{ }^{\circ} \mathrm{C}$, detector temperature $=240{ }^{\circ} \mathrm{C}$, inlet pressure $=10.5 \mathrm{psi} ; \mathrm{t}_{\text {major }}=13.9 \mathrm{~min}, \mathrm{t}_{\mathrm{minor}}=14.3 \mathrm{~min}$.

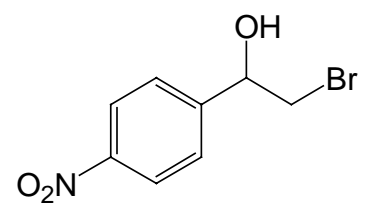

(S)-2-Bromo-1-(4-nitrophenyl)ethanol (21d): $[\alpha]_{\mathrm{D}}^{23}+29.6^{\circ}\left(c\right.$ 1.11, $\left.\mathrm{CHCl}_{3}\right)$; 90.0\% ee. ${ }^{1} \mathrm{H}-\mathrm{NMR}\left(\mathrm{CDCl}_{3}, 300 \mathrm{MHz}\right), \delta .8 .24(\mathrm{~d}, J=8.7 \mathrm{~Hz}, 2 \mathrm{H}), 7.60(\mathrm{~d}, J=8.7$ $\mathrm{Hz}, 2 \mathrm{H})$, 5.08-5.05 $(\mathrm{m}, 1 \mathrm{H}), 3.71-3.66(\mathrm{~m}, 1 \mathrm{H}), 3.57-3.51(\mathrm{~m}, 1 \mathrm{H}), 2.93(\mathrm{br}, 1 \mathrm{H}) \mathrm{ppm}$; GC analysis: CP-cyclodex B-236 M, $0.25 \mathrm{~mm} \times 25 \mathrm{~m}$, column temperature $=145^{\circ} \mathrm{C}$, hold 14 minutes, $10{ }^{\circ} \mathrm{C} /$ minute to $200{ }^{\circ} \mathrm{C}$, hold 20 minute, inject temperature $=240{ }^{\circ} \mathrm{C}$, detector temperature $=260{ }^{\circ} \mathrm{C}$, inlet pressure $=$ $11.5 \mathrm{psi} ; \mathrm{t}_{\text {major }}=32.2 \mathrm{~min}, \mathrm{t}_{\text {minor }}=32.7 \mathrm{~min}$. 
$\overbrace{\mathrm{NO}_{2}}^{\mathrm{OH}} \mathrm{Br}$

(S)-2-Bromo-1-(4-benzyloxy-3-nitrophenyl)ethanol $(21 \mathrm{e}):[\alpha]_{\mathrm{D}}^{23}+31.5^{\circ}(c$ $\left.1.10, \mathrm{CHCl}_{3}\right) ; 93.1 \%$ ee. ${ }^{1} \mathrm{H}-\mathrm{NMR}\left(\mathrm{CDCl}_{3}, 300 \mathrm{MHz}\right), \delta .7 .90(\mathrm{~d}, J=2.2 \mathrm{~Hz}, 1 \mathrm{H})$, 7.55-7.33 (m, 6H), $7.12(\mathrm{~d}, J=8.7 \mathrm{~Hz}, 1 \mathrm{H}), 5.25(\mathrm{~s}, 2 \mathrm{H}), 4.92(\mathrm{dd}, J=3.5,8.7 \mathrm{~Hz}$, $1 \mathrm{H}), 3.62(\mathrm{dd}, J=3.5,10.6 \mathrm{~Hz}, 1 \mathrm{H}), 3.50(\mathrm{dd}, J=8.5,10.5 \mathrm{~Hz}, 1 \mathrm{H}), 2.70(\mathrm{br}, 1 \mathrm{H}) \mathrm{ppm}$; HPLC analysis: Chiralcel OJ, $i-\mathrm{PrOH} / n$-hexane $=35 / 65(\mathrm{v} / \mathrm{v}), 1.0 \mathrm{~mL} / \mathrm{min}, 23{ }^{\circ} \mathrm{C}, \mathrm{UV} 254 \mathrm{~nm} ; \mathrm{t}_{\text {minor }}=16.4$ min, $\mathrm{t}_{\text {major }}=$ $18.9 \min$.

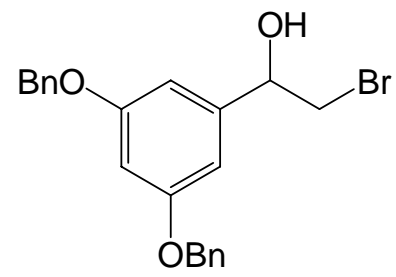

2-Bromo-1-(3,5-bisbenzyloxyphenyl)ethanol (21f): White solid; mp: $85-86{ }^{\circ} \mathrm{C}$; $[\alpha]_{\mathrm{D}}^{23}+20.9^{\circ}\left(c\right.$ 1.00, $\left.\mathrm{CHCl}_{3}\right) ; 96.9 \%$ ee. ${ }^{1} \mathrm{H}-\mathrm{NMR}\left(\mathrm{CDCl}_{3}, 300 \mathrm{MHz}\right), \delta$ 7.44-7.33 (m, 10H), 6.65-6.64 (m, 2H), 6.59-6.57 (m, 1H), $5.04(\mathrm{~s}, 4 \mathrm{H})$, 4.87-4.84 (m, 1H), 3.65-3.60 (m, 1H), 3.51-3.49 (m, 1H), $2.60(\mathrm{br}, 1 \mathrm{H}) \mathrm{ppm} ;{ }^{13} \mathrm{C}-\mathrm{NMR}\left(\mathrm{CDCl}_{3}, 75\right.$ MHz), $\delta: 160.2,142.7,136.6,128.6,128.1,127.5,105.1,102.0,73.8,70.2,40.1$ ppm. IR (KBr) v 3362, 1596, 1438, 1167, 1061, 1042, 731, $693 \mathrm{~cm}^{-1}$. Anal. Calcd for $\mathrm{C}_{22} \mathrm{H}_{21} \mathrm{BrO}_{3}: \mathrm{C}, 63.93 ; \mathrm{H}, 5.12 ; \mathrm{Br}, 19.33$.

Found: $\mathrm{C}, 64.22 ; \mathrm{H}, 5.35 ; \mathrm{Br}, 19.13$. GC analysis: CP-Chirasil-DEX CB, $0.25 \mathrm{~mm} \times 25 \mathrm{~m}$; column temperature $=180{ }^{\circ} \mathrm{C}$, inject temperature $=240{ }^{\circ} \mathrm{C}$, detector temperature $=260{ }^{\circ} \mathrm{C}$, inlet pressure $=10 \mathrm{psi}$; $\mathrm{t}_{\text {minor }}=23.5 \mathrm{~min}, \mathrm{t}_{\text {major }}=30.4 \mathrm{~min}$.

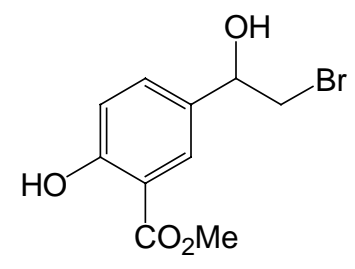

Methyl 5-(2-bromo-1-hydroxyethyl)-2-hydroxybenzoate (21g): White solid; mp: $73-74{ }^{\circ} \mathrm{C} ;[\alpha]_{\mathrm{D}}{ }^{23}+42.1^{\circ}\left(c 1.16, \mathrm{CHCl}_{3}\right) ; 99.3 \%$ ee. ${ }^{1} \mathrm{H}-\mathrm{NMR}\left(\mathrm{CDCl}_{3}, 300\right.$ MHz), $\delta .10 .7(\mathrm{~s}, 1 \mathrm{H}), 7.82(\mathrm{~d}, J=2.3 \mathrm{~Hz}, 1 \mathrm{H}), 7.43(\mathrm{dd}, J=2.3,8.6 \mathrm{~Hz}, 1 \mathrm{H}), 6.95$ $(\mathrm{d}, J=8.6 \mathrm{~Hz}, 1 \mathrm{H}), 4.83(\mathrm{dd}, J=3.6,8.6 \mathrm{~Hz}, 1 \mathrm{H}), 3.93(\mathrm{~s}, 3 \mathrm{H}), 3.56(\mathrm{dd}, J=3.7,10.5 \mathrm{~Hz}, 1 \mathrm{H}), 3.48(\mathrm{dd}$, 
$J=8.6,10.4 \mathrm{~Hz}, 1 \mathrm{H}), 2.94(\mathrm{br}, 1 \mathrm{H}) \mathrm{ppm} ;{ }^{13} \mathrm{C}-\mathrm{NMR}\left(\mathrm{CDCl}_{3}, 75 \mathrm{MHz}\right), \delta .170 .2,161.5,133.3,131.1$, 127.5, 118.0, 112.3, 73.0, 52.4, 40.0 ppm. IR (KBr) v 3507, 2953, 1675, 1615, 1492, 1444, 1330, 1208, 1090, $794 \mathrm{~cm}^{-1}$. Anal. Calcd for $\mathrm{C}_{10} \mathrm{H}_{11} \mathrm{BrO}_{4}$ : C, 43.66; H, 4.03; Br, 29.05. Found: $\mathrm{C}, 43.42 ; \mathrm{H}, 4.02 ; \mathrm{Br}$, 28.85. GC analysis: CP-Chirasil-DEX CB, $0.25 \mathrm{~mm} \times 25 \mathrm{~m}$; column temperature $=180{ }^{\circ} \mathrm{C}$, inject temperature $=240{ }^{\circ} \mathrm{C}$, detector temperature $=260{ }^{\circ} \mathrm{C}$, inlet pressure $=10 \mathrm{psi} ; \mathrm{t}_{\text {minor }}=16.9$ min, $\mathrm{t}_{\text {major }}=$ $17.3 \mathrm{~min}$.

\section{References:}

[1] a) King, L. C.; Ostrum, G. K. J. Org. Chem. 1964, 29, 3459. b) Mehta, N. B.; Musso, D. L. J. Pharm. Soc. 1986, 75, 410.

[2] Haack, K. J.; Hashiguchi, S.; Fujii, A.; Ikariya, T.; Noyori, R. Angew. Chem., Int. Ed. 1997, 36, 285. 